\title{
FGF21 regulates melanogenesis in alpaca melanocytes via ERK1/2-Mediated MITF downregulation
}

Ruiwei Wang ${ }^{1}$, Tianzhi Chen ${ }^{1}$, Bingling Zhao ${ }^{1}$, Ruiwen Fan ${ }^{1}$, Kaiyuan Ji ${ }^{1}$, Xiuju Yu

${ }^{1}$, Xianjun Wang ${ }^{2}$ and Changsheng Dong ${ }^{1, *}$

1 College of Animal Science and Veterinary Medicine, Shanxi Agricultural University, Taigu, 030801, Shanxi

2 Shanxi Normal University, Linfen, 04100, China

* Correspondence: cs_dong@sxau.edu.cn; Tel.: +86-354-628-8208; Fax: $+86-354-622-2942$

Abstract: Fibroblast growth factor 21 (FGF21) is known as a metabolic regulator to regulate the metabolism of glucose and lipids. However, the underlying mechanism of FGF21 on melanin synthesis remains unknown. Therefore, the current study investigates the effect of FGF21 on melanogenesis in alpaca melanocytes. We transfected the FGF21 into alpaca melanocytes, then detected the melanin contents, protein and mRNA levels of pigmentation-related genes in order to determine the melanogenesis-regulating pathway of FGF21. The results showed that FGF21 overexpression suppressed melanogenesis and decreased the expression of the major target genes termed microphthalmia-associated transcription factor (MITF) and its downstream genes, including tyrosinase (TYR) and tyrosinase-related protein 2 (TRP2). However FGF21 increased the expression of phospho-extracellular signal-regulated kinase (p-Erk1/2). In contrast, FGF21-siRNA, a small interference RNA mediating FGF21 silencing, abolished the inhibition of melanogenesis. Altogether, FGF21 may decrease melanogenesis in alpaca melanocytes via ERK 
activation and subsequent MITF downregulation, which is then followed by the suppression of melanogenic enzymes and melanin production.

Keywords: FGF21; melanogenesis; MITF; ERK; FGF21-siRNA, melanocytes

\section{Introduction}

Melanin, synthesized by melanocytes, determines the color of fur, hair and skin.

Melanin containing melanocyte-specific organelles, known as melanosomes, were transfered from melanocytes to adjacent keratinocytes, which formed the distribution of pigmentation and eventually variability of visible colors ${ }^{[1]}$. The melanins are produced in melanocytes and have two main types: brown/black, eumelanin, and red/yellow, pheomelanin ${ }^{[2]}$. It has been confirmed that the melanin synthesis pathway is closely related to melanocyte-specific enzymes - tyrosinase, tyrosinase-related protein 1 , and tyrosinase-related protein $2^{[3]}$. Among these enzymes, tyrosinase is the first rate-limiting enzyme ${ }^{[4]}$. The upregulation of tyrosinase contributes to the formation of eumelanin ${ }^{[5]}$, whereas its downregulation produces pheomelanin ${ }^{[6]}$. The transcriptional regulation of the three major melanogenic enzymes are regulated by $\operatorname{MITF}^{[7]}$, which acts as a central role for melanocyte development, survival, proliferation and melanogenesis ${ }^{[8]}$.

FGF21 showed an abnormal expression in murine melanoma cells ${ }^{[9] .}$ Thus the current study looks at whether or not FGF21 is involved in melanogenesis. MITF expression was inhibited by activating FGF/FGFR/MEK/ERK signaling pathways ${ }^{[10]}$. FGF21 is a new number of the FGF super family ${ }^{[11]}$. This study investigated if FGF21 would also similar to FGFR/MEK/ERK signaling pathways, which would lead 
to MITF downregulation. A previous study showed that FGF receptor mechanisms mediate FGF21 signal pathways ${ }^{[12]}$, which results in FGF21-FGFR complex induced ERK cascade and sustained activation of ERK ${ }^{[13]}$. The ERK signaling pathway is involved in melanin synthesis ${ }^{[14]}$, activation of ERK phosphorylates MITF at serine $73^{[15]}$, followed by degradation of $\mathrm{MITF}^{[16]}$. Therefore, FGF21 might control melanogenesis of alpaca melanocytes via ERK1/2 signal pathway. In this study, FGF21 was selected as a candidate gene for melanogenesis regulation.

\section{Results}

2.1. Effects of FGF21 on melanogenesis in alpaca melanocytes

To detect the effect of FGF21 on melanogenesis in aplaca melanocytes, alpaca melanocytes were transfected with pMSCV-GFP vector encoding FGF21 and FGF21-siRNA. There are two experiments. The first looks at pMSCV-GFP-FGF21 and the pMSCV-GFP control group. The second looks at FGF21-siRNA and the corresponding control group. The qRT-PCR and western blot results showed that the relative mRNA and protein levels of FGF21 in the PMSCV-FGF21 group were 17.58 and 1.82 times higher than that in the control group, respectively (Figure 1A-C). However, in the FGF21-siRNA transfected group, the expression of FGF21 reduced by 4.10 times and 0.62 times for mRNA and protein levels compared with its control group, respectively (Figure 2D-F). These results showed that pMSCV-FGF21 and FGF21-pMSCV has been transfected into alpaca melanocytes efficiently.

To confirm the effect of FGF21 on melanogenesis in aplaca melanocytes, the amount of melanin contents were measured. As shown in Figure 1G, the melanin 
contents decreased significantly in alpaca melanocytes transfected with pMSCV-FGF21-GFP compared to pMSCV-GFP group, while alpaca melanocytes transfected with FGF21-siRNA produced more melanin contents (Figure 1H).

A

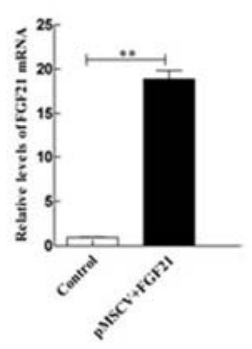

D

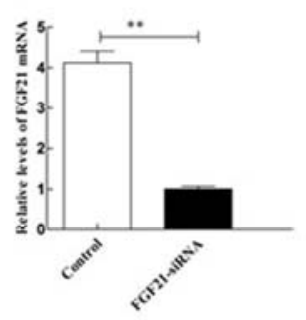

G

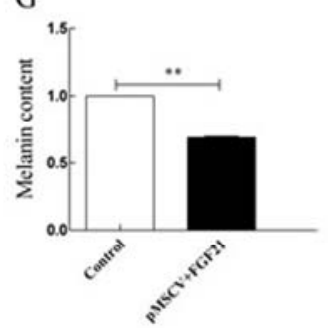

B

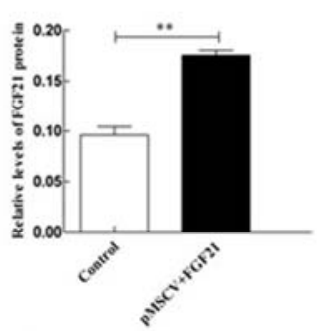

E
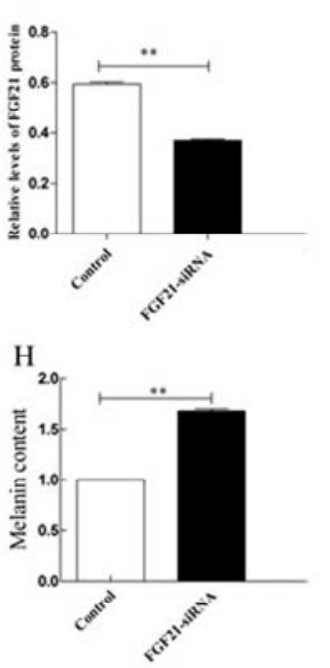

$\mathrm{C}$
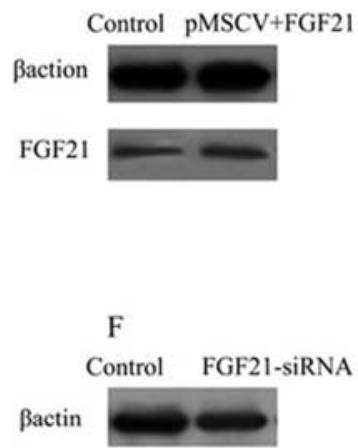

FGF21

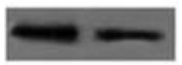

Figure 1. Effect of FGF21 on melanogenesis in alpaca melanocytes. Cells were transfected with pMSCV-FGF21 and FGF21-siRNA as well as their corresponding controls. (A-F) The expression levels of FGF21 were analyzed by qRT-PCR and western blot. (G), (H) Melanin contents were measured, as described in the Materials and Methods section. Results are shown as the mean $\pm \operatorname{SD}(n=3$ each), $* * p<0.01$. 2.2. FGF21 induces MITF downregulation and activates Erk1/2 signaling pathways 
In alpaca melanocytes, overexpression of FGF21 reduced melanin content, thus FGF21 may have effects on MITF expression, which functions as a key regulator on melanogenesis ${ }^{[17]}$.As shown in Figure $2 \mathrm{~A}-\mathrm{C}, \mathrm{qRT}-\mathrm{PCR}$ and western blotting results showed that, for the FGF21-transfected group, the relative level of MITF were substantially lower for mRNA and protein when compared with the control group.

Previous studies have demonstrated that sustained phosphorylation of ERK degrades MITF and subsequent MITF phosphorylation ${ }^{[18]}$. Therefore, this study will be verified if FGF21 activates ERK signaling pathways. The qRT-PCR and western blot results showed that FGF21 stimulated the ERK1/2 signaling pathway. Moreover, the phosphorylation of MITF was associated with activation of ERK, leading to downregulation of TYR, TRP1 and TRP2 ${ }^{[19]}$. In this study, the target genes of MITF and key melanic enzymes TYR and TRP2 for melanin production were also reduced by different amounts (Figure 2A-C). The results supported that FGF21 overexpression induced ERK signaling pathways and downregulation of MITF, TYR and TRP2, which resulted in a reduction of melanin contents. 


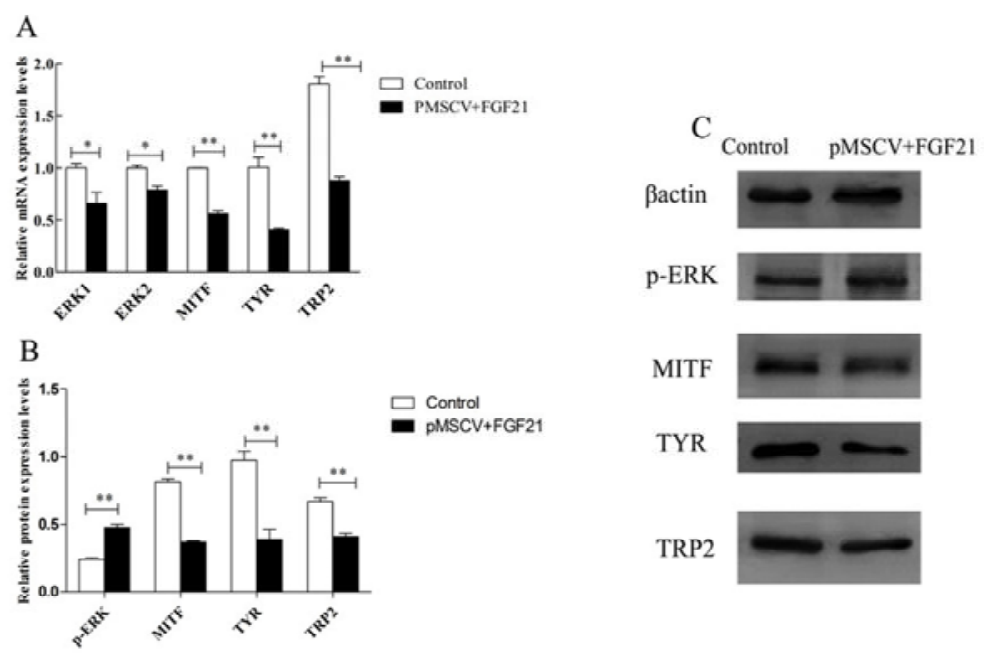

Figure 2. FGF21 overexpression affects melanin synthesis in alpaca melanocytes. The pMSCV-FGF21 and pMSCV-GFP was transfected into the alpaca melanocytes effectively. Total RNA and protein was extracted for the analysis of qRT-PCR and western blot. (A) The mRNA expression of ERK1/2, MITF, TYR, TRP2 was amplified with specific primers and measured by qRT-PCR, and $18 \mathrm{~S}$ was used as an mRNA loading control. (B), (C) Western blot of cells for relative protein abundance with antibodies recognized phospho-specific ERK, MITF, TYR and TRP2. Protein loading was normalized relative to $\beta$-actin. The results are shown as the mean $\pm \operatorname{SD}(n$ $=3$ each $) * p<0.05, * * p<0.01$

2.3. FGF21-siRNA abolishes hypopigmentation via inhibiting ERK1/2 signaling pathway

The effect of FGF21-siRNA on ERK1/2 signaling and melanogenesis was further investigated. FGF21-siRNA abrogated the activation of ERK1/2 induced by FGF21 was found. A previous study showed that ERK1/2 inhibitor lead to the activation of melanin synthesis ${ }^{[20]}$. In this study, the relative expression of melanin contents in 
FGF21-siRNA-treated melanocytes increased significantly when compared with the control group. Collectively, the expression of MITF, TYR and TRP2 reversed at different levels after FGF21-siRNA transfection. The results showed that FGF21-siRNA may abolish hypopigmentation via inhibiting ERK1/2 signaling pathway.
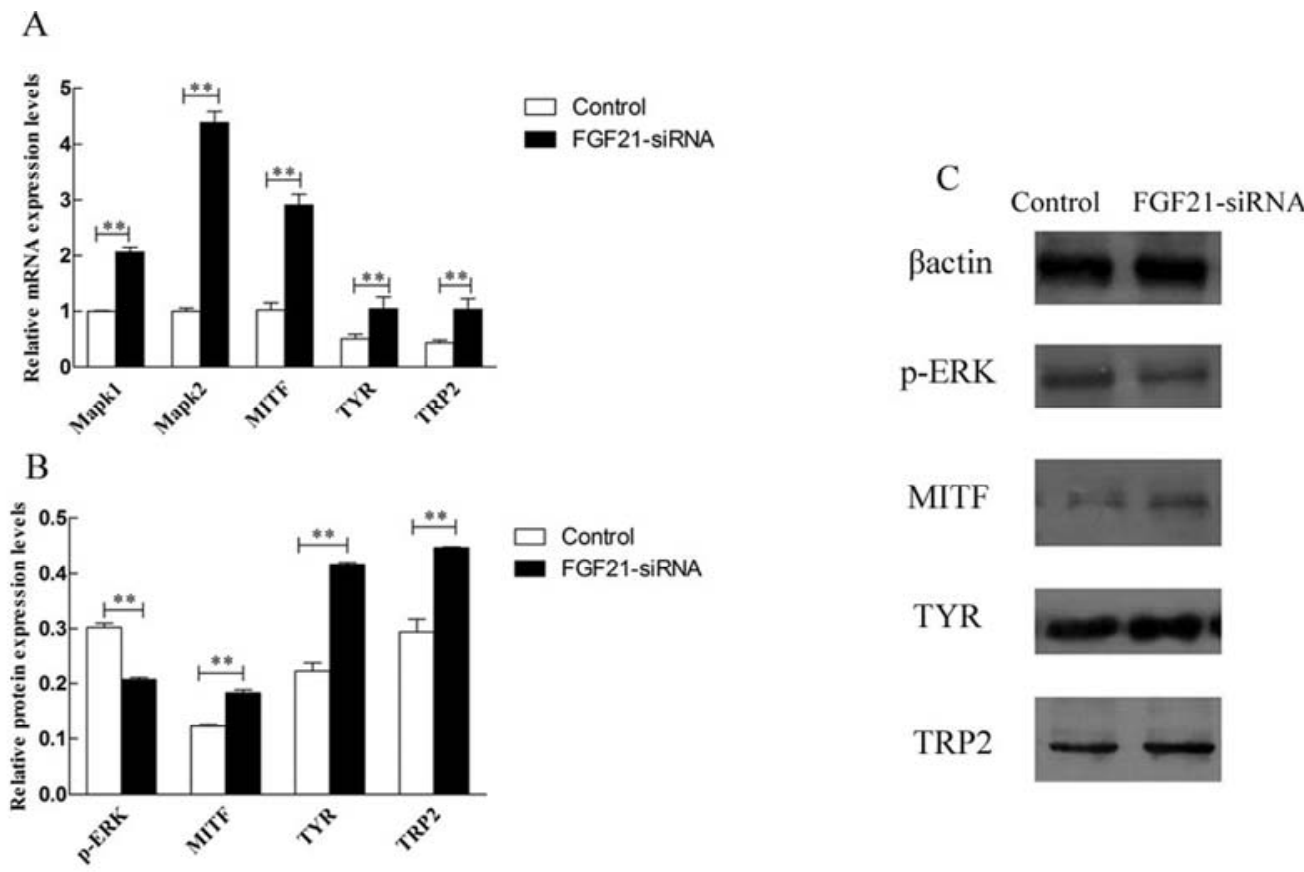

Figure 3. Effects of FGF21-siRNA on ERK1/2 pathway and melanogenesis-related genes. (A) The mRNA expression of FGF21 and melanogenesis-related genes were analyzed by qRT-PCR. (B) The protein expression of FGF21 and melanogenesis-related genes were analyzed by western blot. The results are shown as the mean $\pm \mathrm{SD}(n=3$ each $),{ }^{*} p<0.01$.

\section{Discussion}

Melanin is actually an indole polymer originated from the oxidation of tyrosine and tyrosinase-related enzymes ${ }^{[21]}$. TYR oxidizes L-tyrosine to 3,4-dihydroxy 
phenylalanine (DOPA) and then the oxidation of ODPA to dopaquinone ${ }^{[22]}$. TRP2, the dopachrome delta-isomerase (DCT), promotes DC to DHTCA ${ }^{[23]}$. With the catalysis of TRP1, eumelanin monomer named 5,6-dihydroxyindole-2-carboxylic acid (DHICA) composed eumelanin polymer ${ }^{[24]}$. MITF is a basic helix-loop-helix-leucine zipper (bHLH-LZ) transcription factor ${ }^{[25,26]}$, which regulates melanogenesis by binding to Mand E-box's promoter of TYR, TRP1 and TRP2 ${ }^{[27,28]}$. MITF is subjected to complex regulatory controls by multiple signal pathways, such as Wnt signaling pathway, cAMP pathway, extracellular signal-regulated kinase1/2 (ERK1/2) pathway, NO-mediated pathway regulated MITF at transcriptional or post-transcriptional levels ${ }^{[29]}$. ERK signaling cascade is one of the classic signal pathways, having an essential role in cell growth, development, proliferation and differentiation ${ }^{[30]}$. Increased activation of ERK pathway stimulated MITF for ubiquitin-dependent $\operatorname{degradation}^{[31]}$.

More and more biological functions of FGF21 were exhibited, but a comprehensive understanding of FGF21 on melanogenesis has been lacking. Several studies have shown that FGF21 could activate ERK1/2 pathways $^{[32] .}$ After alpaca melanocytes were transfected with PMSCV-FGF21, the protein, phospho-ERK1/2, increased and the mRNA, ERK1/2, decreased. The results show that FGF21 stimulates phosphorylation of Erk1/2 and activates Erk1/2 signal pathways. Previous studies have reported that phospho-Erk1/2 targets MITF phosphorylation and ubiquitin-dependent proteolysis ${ }^{[33]}$. The activation of ERK also had inhibitory effect on tyrosinase activity and transcription ${ }^{[34,35]}$. As expected, FGF21 decreased 
melanogenesis by a downregulation of MITF, TYR, TRP2, which might be associated with ERK activation.

However, the inhibition of ERK stimulated melanin synthesis and tyrosinase activity $^{[36]}$. Alpaca melanocytes treated with FGF21-siRNA and its corresponding control group reversed the inital inhibitory effect of FGF21 on MITF and tyrosinase enzymes,. The augmented melanin pigmentation associated with upregulation of MITF is mediated by the inhibition of the ERK1/2 pathway.

\section{Materials and Methods}

\subsection{Antibodies}

Antibodies recognizing phospho-specific ERK1/2 (Thr202/Tyr204) were purchased from Cell Signaling Technology (Danvers, MA, USA). FGF21, MITF, TYR, TYRP2 were obtained from Abcam Biotechnology (Abcam, Cambridge, UK). $\beta$-action was obtained from CW Biotech.

\subsection{Cell cultures And Cell transfection}

All alpaca melanocytes used in this study were established in Laboratories of Alpaca Biology, College of Animal Science and Technology, Shanxi Agricultural University, China. Alpaca melanocytes were cultured in Melanocyte Medium (MelM) (ScienCell Research Laboratories, Carlsbad, CA, USA), and were supplemented with 1\% Melanocyte growth supplement (MELGS), $0.5 \%$ fetal bovine serum(FBS), 100u/ml Penicillin and $100 \mathrm{ug} / \mathrm{ml} \mathrm{Streptomycin} \mathrm{at} 37 \square$ in a $5 \% \mathrm{CO} 2$-containing atmosphere.

FGF21 was synthesized and cloned into pMSCV-GFP plasmid (Addgene, 
Cambridge, MA, USA), resulting in the introduction of FGF21. FGF21-siRNA was purchased from Sangon Biotech, and mediates gene FGF21 silencing. Alpaca melanocytes were transfected using Lipofectamine 3000 reagent (Invitrogen, USA), according to the operation manual kit. $48 \mathrm{~h}$ later, the melanocytes were harvested.

\subsection{Melanin Content Assay}

Melanin was extracted for a melanin content assay. Melanocytes were harvested and cleaned with cold PBS 3 times. Then cell precipitation was dissolved in 1mol/L $\mathrm{NaOH}$ at $80 \square$ for 30 mins. The relative melanin content (ug/10 $0^{6}$ cells) was measured at $475 \mathrm{~nm}$ using a Microplate Reader. Before measuring, melanocytes were observed and counted.

\subsection{Total RNA Extraction and qRT-PCR Analysis}

Total RNA was isolated from melanocyte cells using TRIzol Reagent (Life Technology). Synthesis of cDNA for real-time PCR analysis of FGF21 expression in cultured melanocytes was performed using the miRNA qRT-PCR kit according to the manufacturer's instructions. The levels of gene mRNA were measured using SYBR®Premix Ex TaqTMII (Tli RNaseH Plus) according to the manufacturer's instructions (TAKARA, Dalian, China).

Conditions for real-time PCR were as follows: preheating at $95 \square$ for $5 \mathrm{~min}$, followed by 40 cycles of shuttle heating at $95 \square$ for 30 s, annealing temperature at $60 \square$ for 30 s, extending temperature at $72 \square$ for 20 s. Quantification of mRNA level for the target gene was performed using the comparative threshold cycle (CT) method. The expression of mRNA for the target genes was normalized relative to the abundance of 18S rRNA. The primer sequences are shown in Table 1. 
Table 1. primer sequences and qRT-PCR production for targeting gene

\begin{tabular}{|c|c|c|}
\hline Primer Name & Primer Sequence 5' $\rightarrow$ 3' & $\begin{array}{c}\text { PCR Production } \\
\text { (bp) }\end{array}$ \\
\hline Alpaca-FGF21-F & CCGGGCTCGAGATGGACTGGGACGAGGCC & \multirow{2}{*}{629} \\
\hline Alpaca- $F G F 21-\mathrm{R}$ & CCGGGGAATTCTCAGGAAGTGTAGCTGGGGC & \\
\hline Alpaca-FGF21-F & CGGGAGTTGCTTCTTGAG & \multirow{2}{*}{116} \\
\hline Alpaca- $F G F 21-\mathrm{R}$ & CACCTCCAAAGCATCCAG & \\
\hline Alpaca-MITF-F & TCCCAAGTCAAATGATCCAG & \multirow{2}{*}{114} \\
\hline Alpaca-MITF-R & GAGCCTGCATTTCAAGTTCC & \\
\hline Alpaca-TRP2-F & TGCTTTGCCCTACTGGAAC & \multirow{2}{*}{112} \\
\hline Alpaca-TRP2-R & ATCAGAGTCGATCGTCTG & \\
\hline Alpaca-Erk1-F & TGCTGCTCAACACCACCT & \multirow{2}{*}{272} \\
\hline Alpaca-Erkl-R & TCCGAGAATACCCAGAATG & \\
\hline Alpaca-Erk2-F & CAGGTTGTTCCCAAATGC & \multirow{2}{*}{196} \\
\hline Alpaca-Erk2-R & CTCCTTAGGTAAGTCGTCCA & \\
\hline Alpaca- $18 S$-F & GAAGGGCACCACCAGGACT & \multirow{2}{*}{158} \\
\hline Alpaca-18S-R & CAGACAAATCACTCCACCAA & \\
\hline
\end{tabular}

\subsection{Western Blotting}

Total protein was extracted from melanocytes using a protein extraction reagent (RIPA Lysis Buffer, Beyotime) according to the manufacturer's guidelines. Concentration was measured using a nucleic acid/protein analyzer. Protein(200ng/lane) was separated using $10 \%$ SDS-PAGE electrophoresis and transferred onto nitrocellulose filter membranes. The membranes were blocked in 5\% skim milk for 1 h. Then incubated membranes were diluted in $1 \times$ TBS with $0.1 \%$ Tween 20 at $4^{\circ} \mathrm{C}$ while being gentle shaken overnight. The next day, the membrane was washed three times with TBST and incubated with diluted HRP-conjugated secondary antibody for 
$1 \mathrm{~h}$ at $37^{\circ} \mathrm{C}$. Finally, the membranes were washed 6 times with TBST for 5 minutes each. The protein abundance was analyzed using Image-Pro Plus Software, version 6.0 (Media Cybernetics), and normalized relative to $\beta$-actin in each lane.

4.6.Statistical analysis

All of the results were repeated in triplicate and expressed as mean \pm standard errors. Each set of data was analyzed by GraphPad Prism 5.0 software (GraphPad Software Inc,CA, USA). P-values $<0.05$ for the experimental and corresponding control groups were considered statistically significant, and p-values $<0.001$ represent highly significant results.

\section{Conclusions}

In this research, we found FGF21 overexpression reduced melanin synthesis via ERK signaling pathway, accompanied with downregulation of MITF and target genes, including TYR and TRP2. In contrast, FGF21-siR stopped hypopigmentation by inhibiting ERK1/2, and the expression of MITF and melanin-specific enzymes increased when compared with the control groups. As a result, FGF21 may decrease melanogenesis of alpaca melanocytes via ERK1/2-Mediated MITF degradation. Altogether, FGF21 may decrease melanogenesis of alpaca melanocytes via ERK1/2-Mediated MITF downregulation.

\section{Acknowledgments}

This research was supported by the High Technology Research and Development Program of China (863 Program) (2013AA102506), the Special Foundation for 
Agro-scientific Research in the Public Interest (201303119) and the Aid Program for

Innovative Research Team in Shanxi Agricultural University (CXTD201201).

\section{References}

1. Hearing $\mathrm{V}$ J Biochemical control of melanogenesis and melanosomal organization. [J]. J Investig Dermatol Symp Proc, 1999, 4(1): 24-28.

2. Lin J Y, Fisher D E. Melanocyte biology and skin pigmentation.[J]. Nature, 2007, 445(7130): 843-50.

3. Rad H H, Yamashita T, Jin H Y, et al. Tyrosinase-related proteins suppress tyrosinase-mediated cell death of melanocytes and melanoma cells. [J]. Experimental Cell Research, 2004, 298(2): 317-28.

4. Schweikardt T, Olivares C, Solano F, et al. A three-dimensional model of mammalian tyrosinase active site accounting for loss of function mutations[J]. Pigment Cell Research, 2007, 20(5): 394-401.

5. Kuzumaki T, Matsuda A, Wakamatsu K, et al. Eumelanin biosynthesis is regulated by coordinate expression of tyrosinase and tyrosinase-related protein-1 genes[J]. Experimental Cell Research, 1993, 207(1): 33.

6. Lamoreux M L, Wakamatsu K, Ito S. Interaction of major coat color gene functions in mice as studied by chemical analysis of eumelanin and pheomelanin[J]. Pigment Cell Research, 2001, 14(1): 23-31.

7. Bentley N J, Eisen T, Goding C R. Melanocyte-specific expression of the human tyrosinase promoter: activation by the microphthalmia gene product and role of the initiator.[J]. Molecular \& Cellular Biology, 1994, 14(14): 7996-8006. 
8. Levy C, Khaled M, Fisher D E. MITF: master regulator of melanocyte development and melanoma oncogene[J]. Trends in Molecular Medicine, 2006, 12(9): 406-414.

9. Osawa T, Muramatsu M, Watanabe M, et al. Hypoxia and low-nutrition double stress induces aggressiveness in a murine model of melanoma[J]. Cancer Science, 2009, 100(5): 844-851.

10. Spence J R, Madhavan M, Aycinena J C, et al. Retina regeneration in the chick embryo is not induced by spontaneous Mitf downregulation but requires FGF/FGFR/MEK/Erk dependent upregulation of Pax6[J]. Molecular Vision, 2007, 13-57.

11. Kharitonenkov A, Shiyanova T L, Koester A, et al. FGF-21 as a novel metabolic regulator.[J]. Journal of Clinical Investigation, 2005, 115(6): 1627-35.

12. Suzuki M, Uehara Y, Motomuramatsuzaka K, et al. betaKlotho is required for fibroblast growth factor (FGF) 21 signaling through FGF receptor (FGFR) 1c and FGFR3c. [J]. Molecular endocrinology (Baltimore, Md.), 2008, 22(4): 1006-14.

13. Lin Z, Tian H, Lam KS, et al. Adiponectin mediates the metabolic effects of FGF21 on glucose homeostasis and insulin sensitivity in mice[J]. Cell Metabolism, 2013, 17(5): 779-89.

14. Kim D S, Hwang E S, Lee J E, et al. Sphingosine-1-phosphate decreases melanin synthesis via sustained ERK activation and subsequent MITF degradation[J]. Journal of Cell Science, 2003, 116(Pt 9): 1699. 
15. Hemesath T J, Price E R, Takemoto C, et al. MAP kinase links the transcription factor Microphthalmia to c-Kit signalling in melanocytes[J]. Nature, 1998, 391(6664): 298-301.

16. Lee H J, Lee W J, Chang S E, et al. Hesperidin, A Popular Antioxidant Inhibits Melanogenesis via Erk1/2 Mediated MITF Degradation[J]. International Journal of Molecular Sciences, 2015, 16(8): 18384-95.

17. Widlund H R, Fisher D E. Microphthalamia-associated transcription factor: a critical regulator of pigment cell development and survival.[J]. Oncogene, 2003, 22(20): 3035-3041.

18. Lee W R, Shen S C, Wu P R, et al. CSE1L Links cAMP/PKA and Ras/ERK pathways and regulates the expressions and phosphorylations of ERK1/2, CREB, and MITF in melanoma cells.[J]. Molecular Carcinogenesis, 2015, 55(11): 1542-1552.

19. Yavuzer U, Keenan E, Lowings P, et al. The Microphthalmia gene product interacts with the retinoblastoma protein in vitro and is a target for deregulation of melanocyte-specific transcription.[J]. Oncogene, 1995, 10(1): 123-34.

20. Donaubauer E M, Law N C, Hunzickerdunn M E. Follicle-Stimulating Hormone (FSH)-dependent Regulation of Extracellular Regulated Kinase (ERK) Phosphorylation by the Mitogen-activated Protein (MAP) Kinase Phosphatase MKP3[J]. Journal of Biological Chemistry, 2016, 291: jbc. M116. 733972.

21. Del M V, Beermann F. Tyrosinase and related proteins in mammalian pigmentation.[J]. Febs Letters, 1996, 381(3): 165-8. 
22. Hearing V J, Ekel T M. Mammalian tyrosinase. A comparison of tyrosine hydroxylation and melanin formation.[J]. Biochemical Journal, 1976, 157(3): 549.

23. Tsukamoto, K.; Jackson, I.J.; Urabe, K.; Montague, P.M.; Hearing, V.J. A second tyrosinase-related protein, TRP-2, is a melanogenic enzyme termed DOPAchrome tautomerase. EMBO J. 1992, 11, 519-526.

24. Kobayashi T, Urabe K, Winder A, et al. Tyrosinase related protein 1 (TRP1) functions as a DHICA oxidase in melanin biosynthesis[J]. Embo Journal, 1994, 13(24): 5818-25.

25. Vetrini, F.; Auricchio, A.; Du, J.; Angeletti, B.; Fisher, D.E.; Ballabio, A.; Marigo, V. The microphthalmia transcription factor (MITF) controls expression of the ocular albinism type 1 gene: Link between melanin synthesis and melanosome biogenesis. Mol. Cell.Biol. 2004, 24, 6550-6559.

26. Hodgkinson C A, Moore K J, Nakayama A, et al. Mutations at the mouse microphthalmia locus are associated with defects in a gene encoding a novel basic-helix-loop-helix-zipper protein[J]. Cell, 1993, 74(2): 395-404.

27. Ganss R, Schütz G, Beermann F. The mouse tyrosinase gene. Promoter modulation by positive and negative regulatory elements[J]. Journal of Biological Chemistry, 1994, 269(47): 29808-16.

28. Dunn A S, Coller B. Regulation of tyrosinase gene expression by cAMP in B16 melanoma cells involves two CATGTG motifs surrounding the TATA box: implication of the microphthalmia gene product.[J]. Journal of Cell Biology, 1996, 134(3): 747-55.

29. Buscà R, Ballotti R. Cyclic AMP a key messenger in the regulation of skin pigmentation[J]. Pigment Cell Research, 2000, 13(2): 60-9. 
30. Marshall C J. Specificity of receptor tyrosine kinase signaling: transient versus sustained extracellular signal-regulated kinase activation.[J]. Cell, 1995, 80(2): $179-85$.

31. Feng L, Singh A, Zhen Y, et al. MiTF links Erk1/2 kinase and p21 CIP1/WAF1, activation after UVC radiation in normal human melanocytes and melanoma cells[J]. Molecular Cancer, 2010, 9(1): 1-12.

32. Fisher F M, Chui P C, Antonellis P J, et al. Obesity Is a Fibroblast Growth Factor 21 (FGF21)-Resistant State[J]. Diabetes, 2010, 59(11): 2781-2789.

33. Alam M, Seo B J, Zhao P, et al. Anti-Melanogenic Activities ofHeracleum moellendorffiivia ERK1/2-Mediated MITF Downregulation: [J]. International Journal of Molecular Sciences, 2016, 17(11): 1844.

34. Son Y, Lee S, Kim S, et al. Acteoside inhibits melanogenesis in B16F10 cells through ERK activation and tyrosinase down-regulation[J]. Journal of Pharmacy \& Pharmacology, 2011, 63(10): 1309-19.

35. Drira R, Sakamoto K. Sakuranetin Induces Melanogenesis in B16BL6 Melanoma Cells through Inhibition of ERK and PI3K/AKT Signaling Pathways. [J]. Phytotherapy Research Ptr, 2016, 30(6): n/a-n/a. 
bioRxiv preprint doi: https://doi.org/10.1101/147082; this version posted June 7, 2017. The copyright holder for this preprint (which was not certified by peer review) is the author/funder. All rights reserved. No reuse allowed without permission. 
A

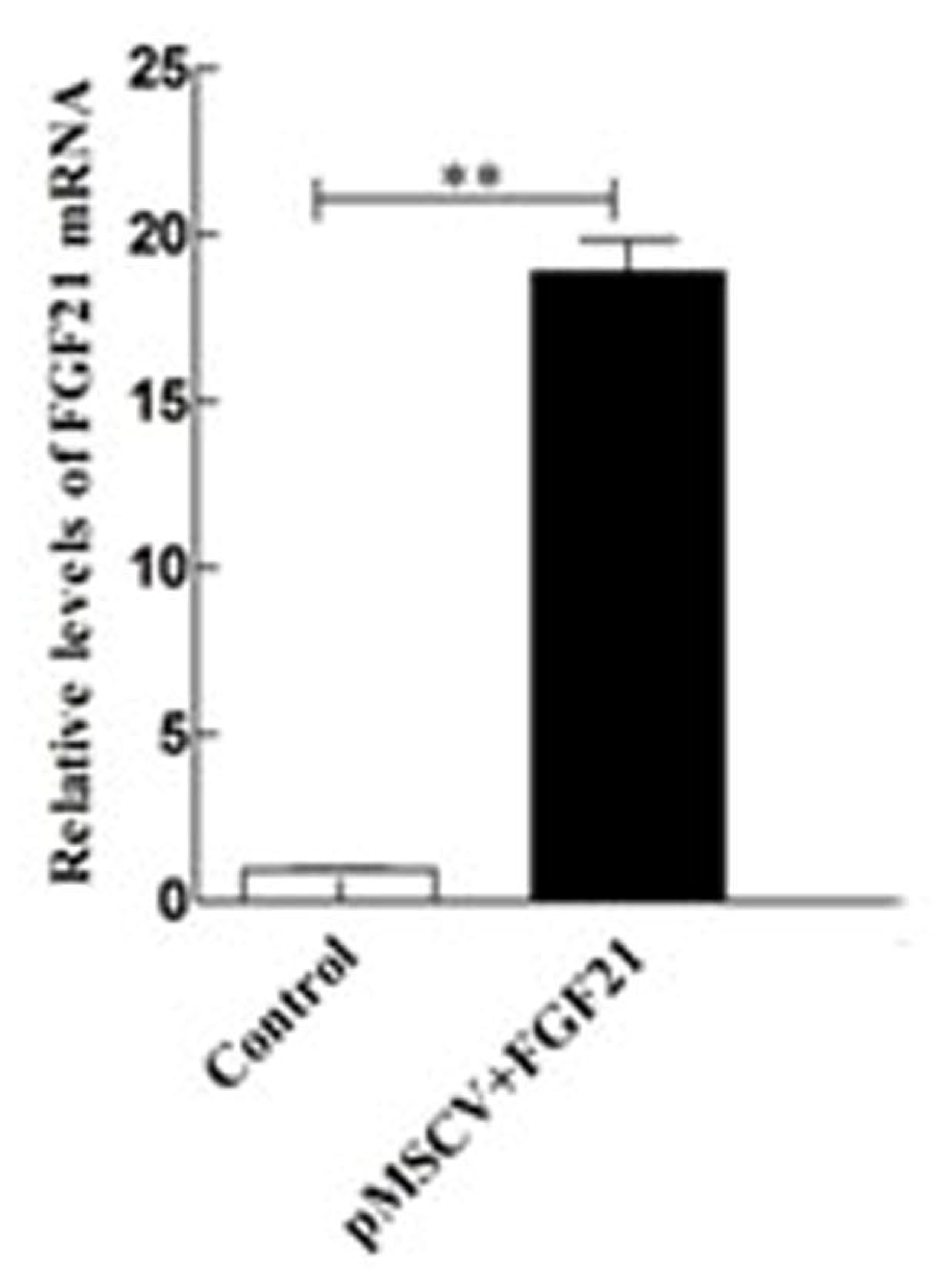

D

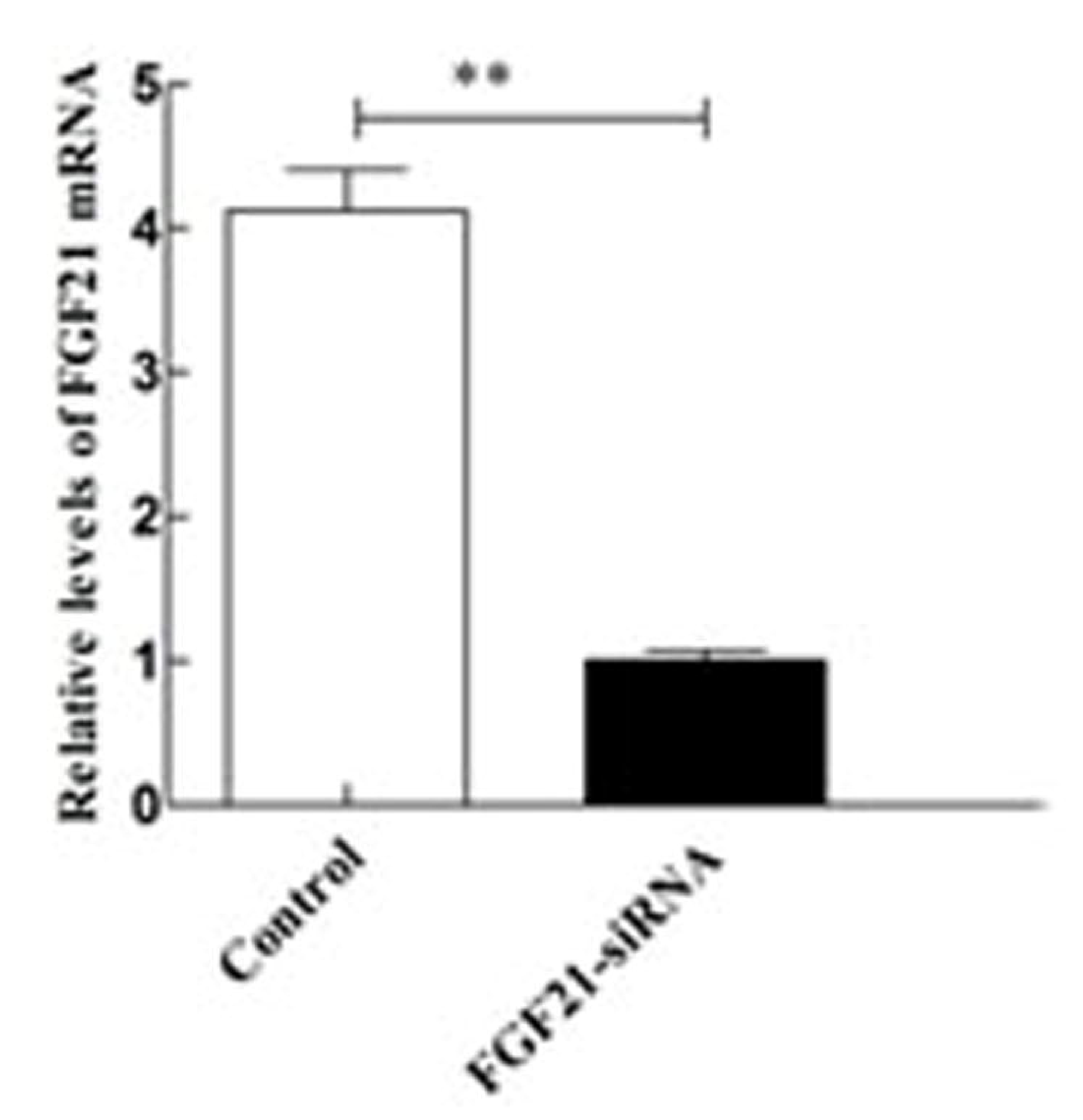

G

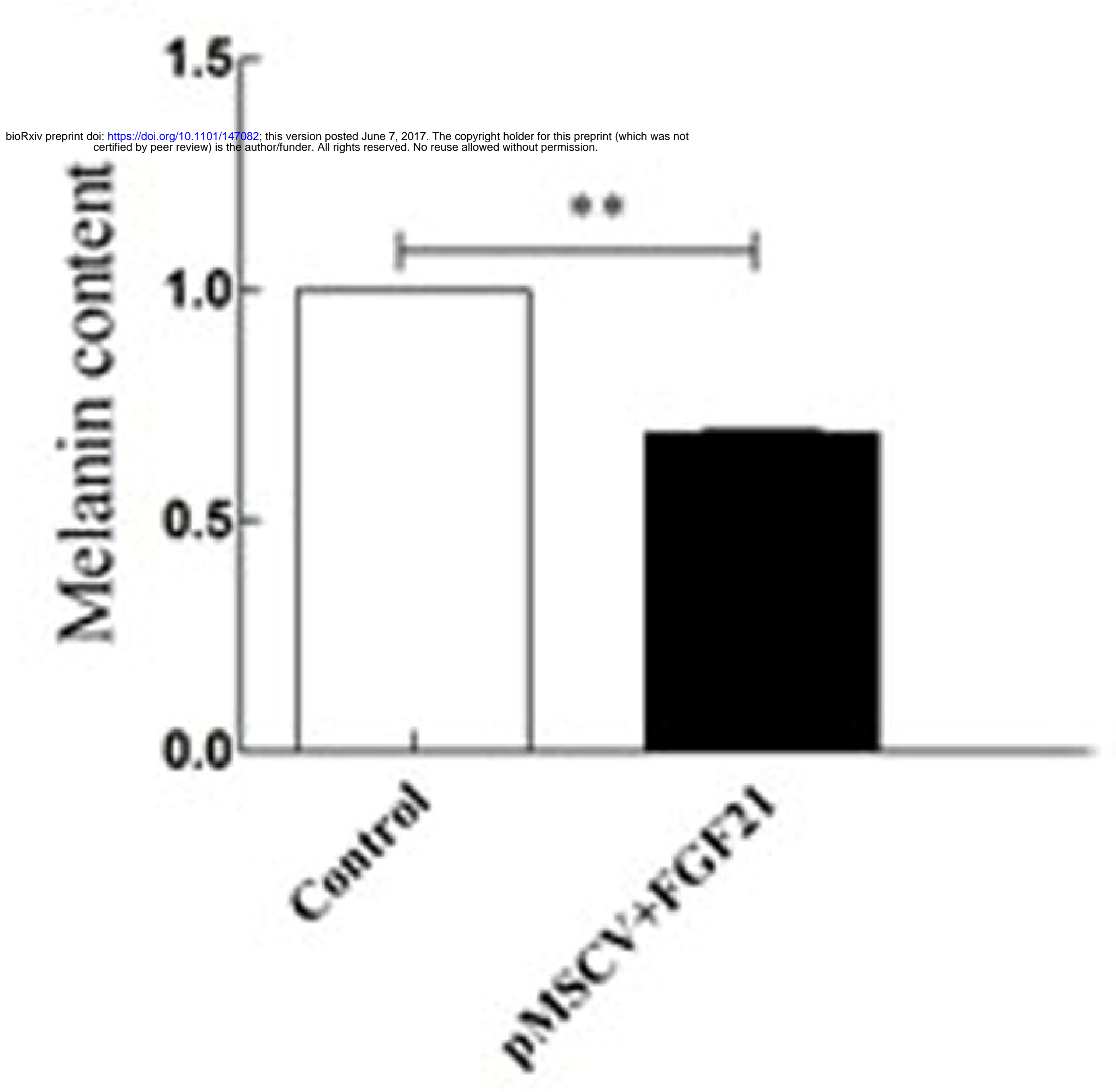

B

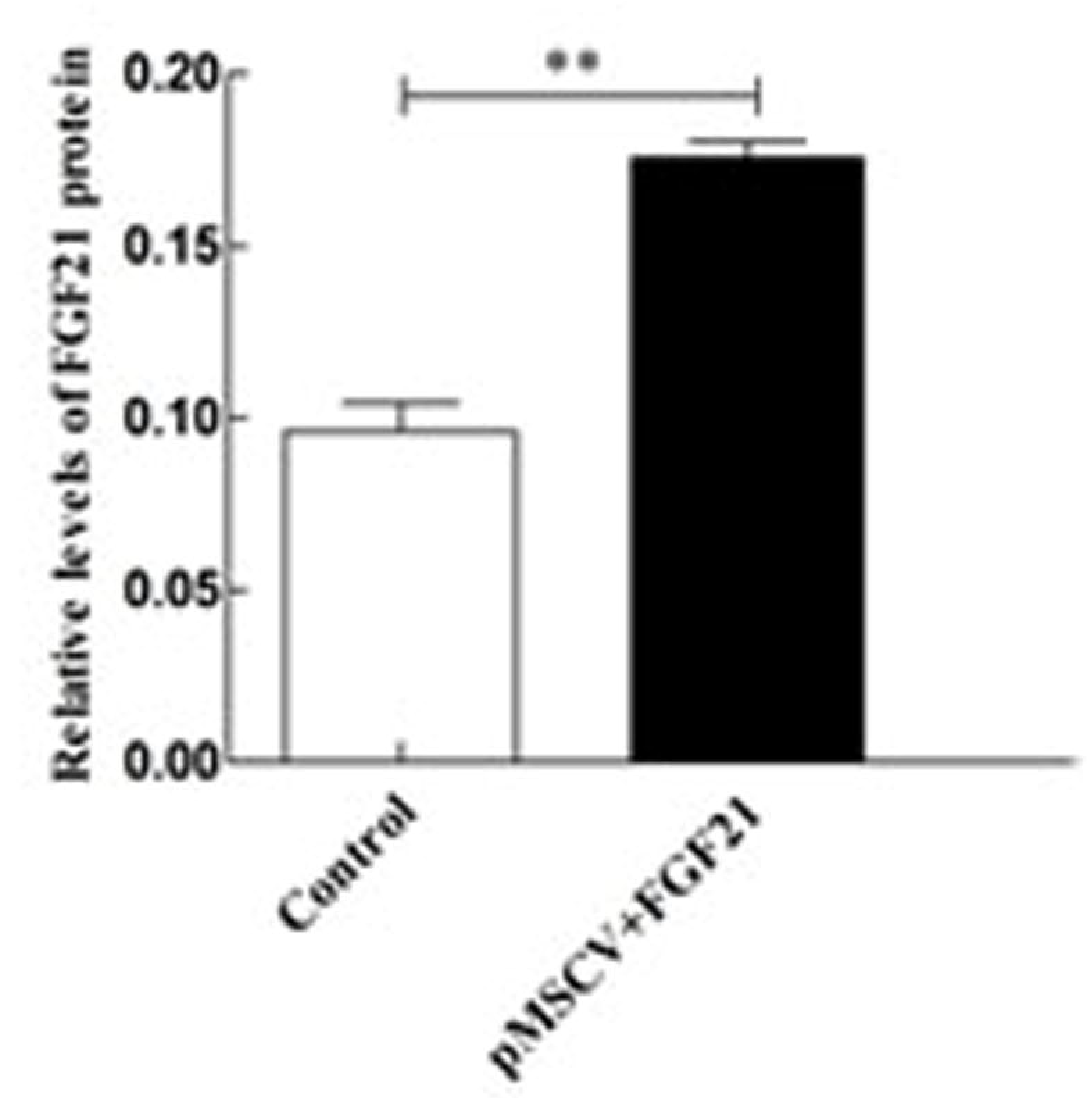

E

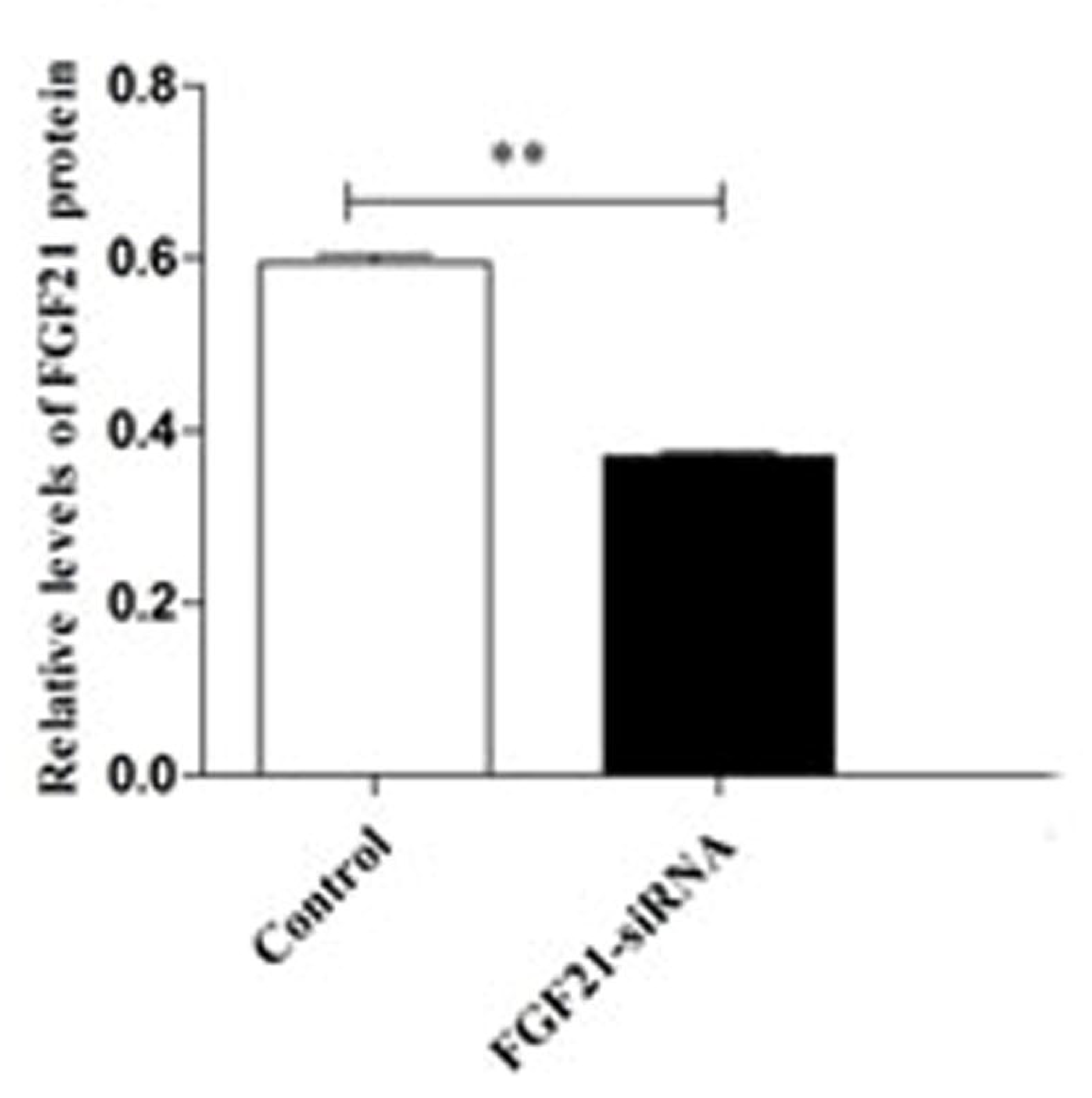

$\mathrm{H}$

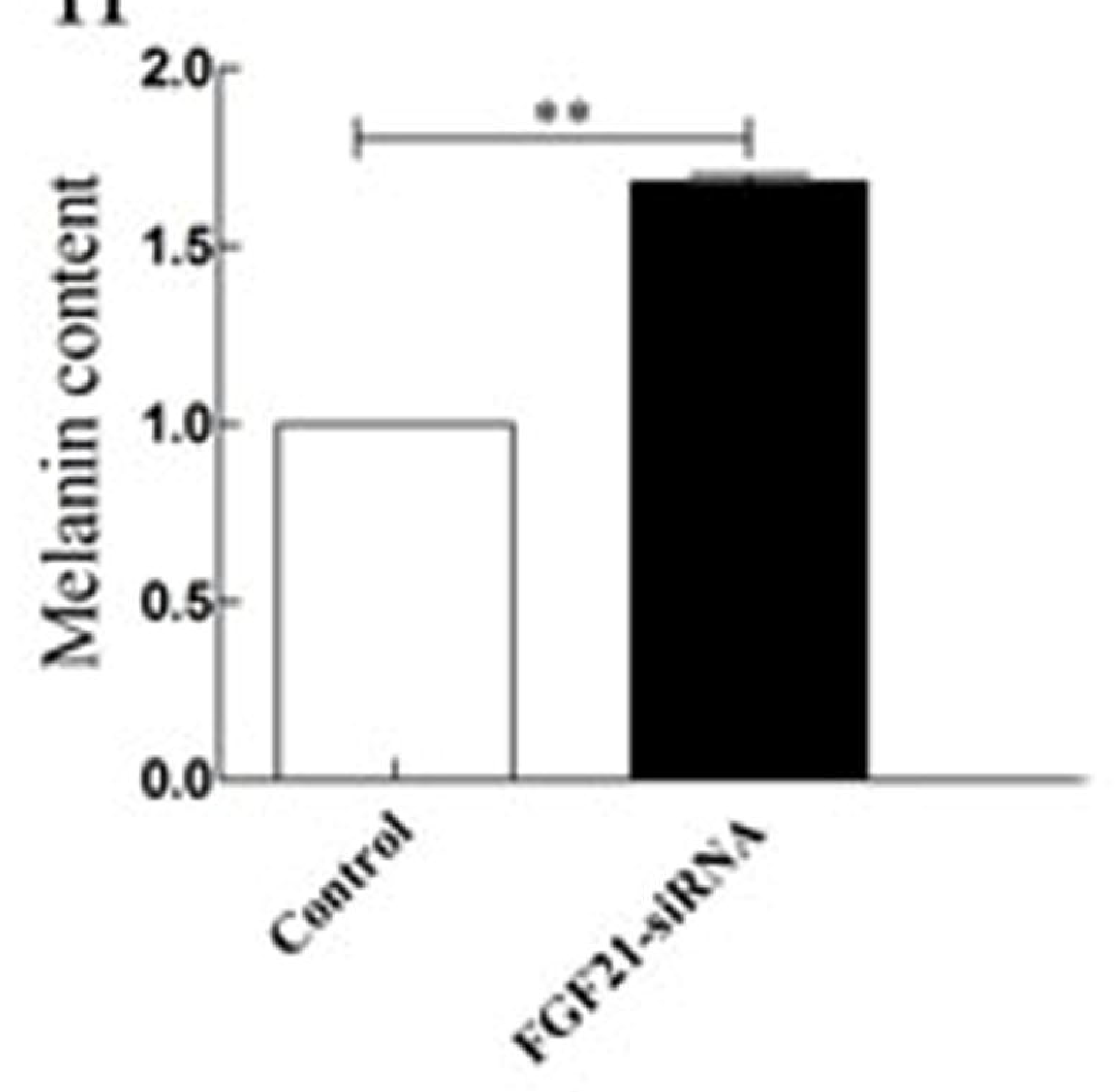

Control pMSCV+FGF21

ßaction

FGF21
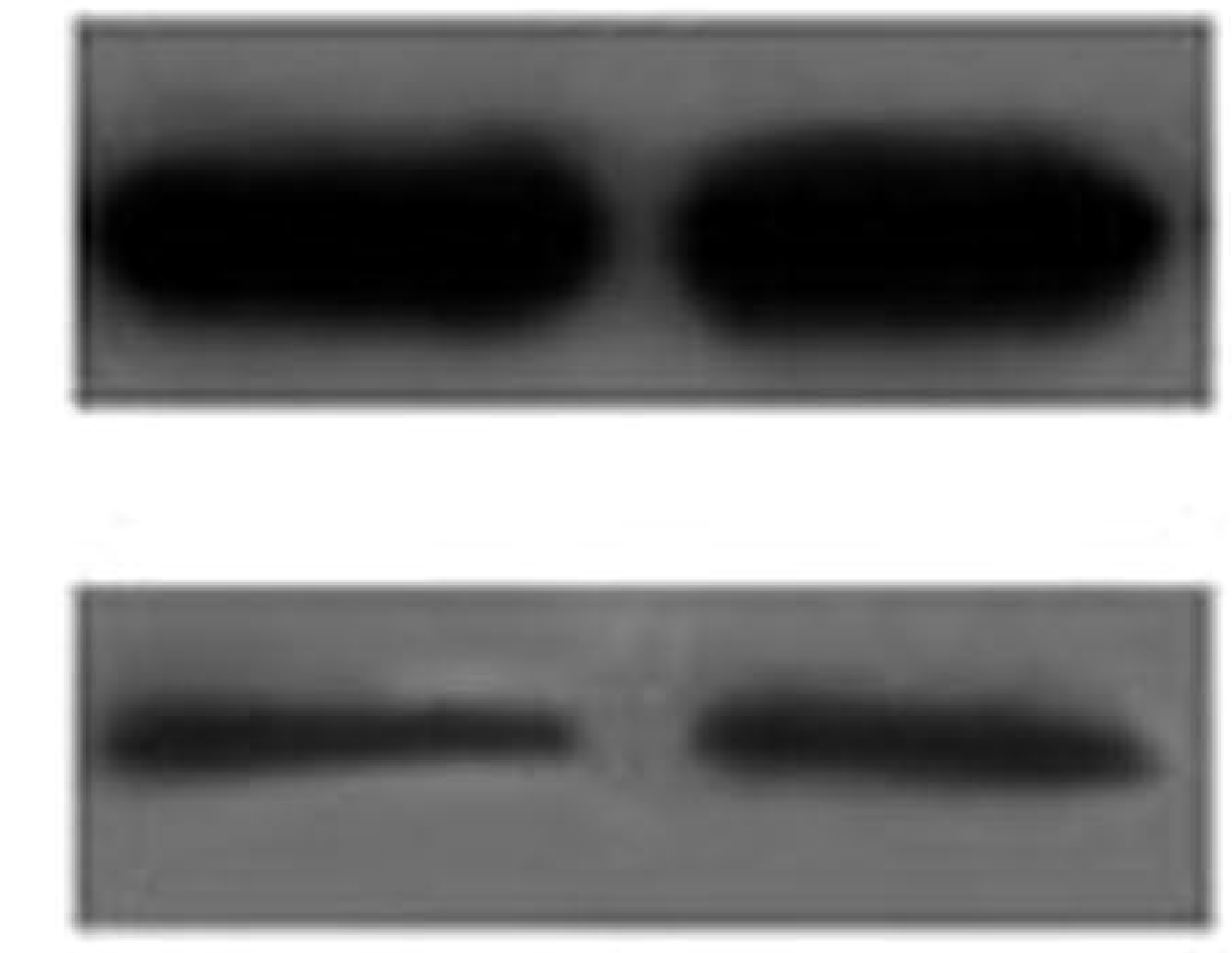

F

Control FGF21-siRNA

Bactin

FGF21
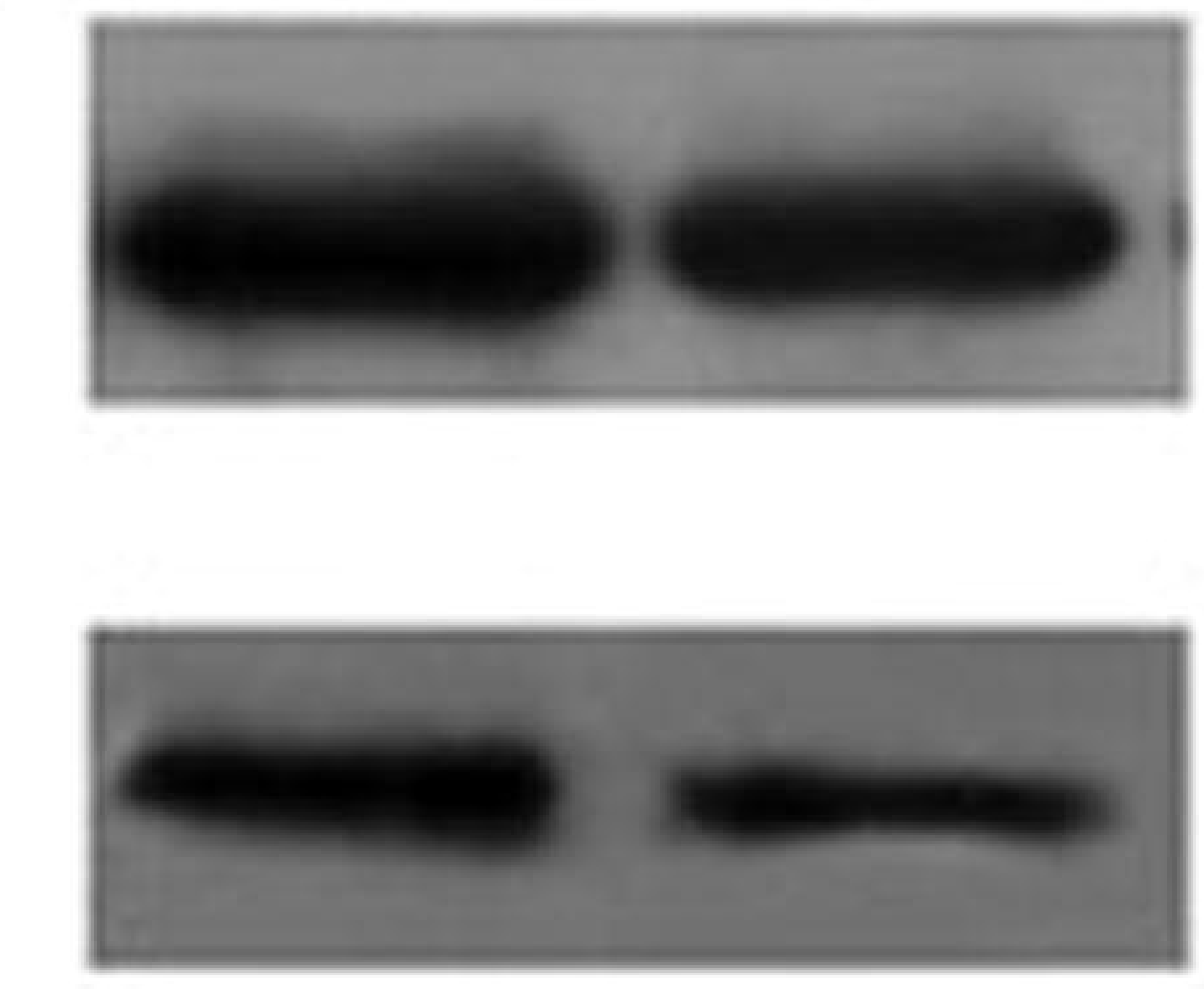

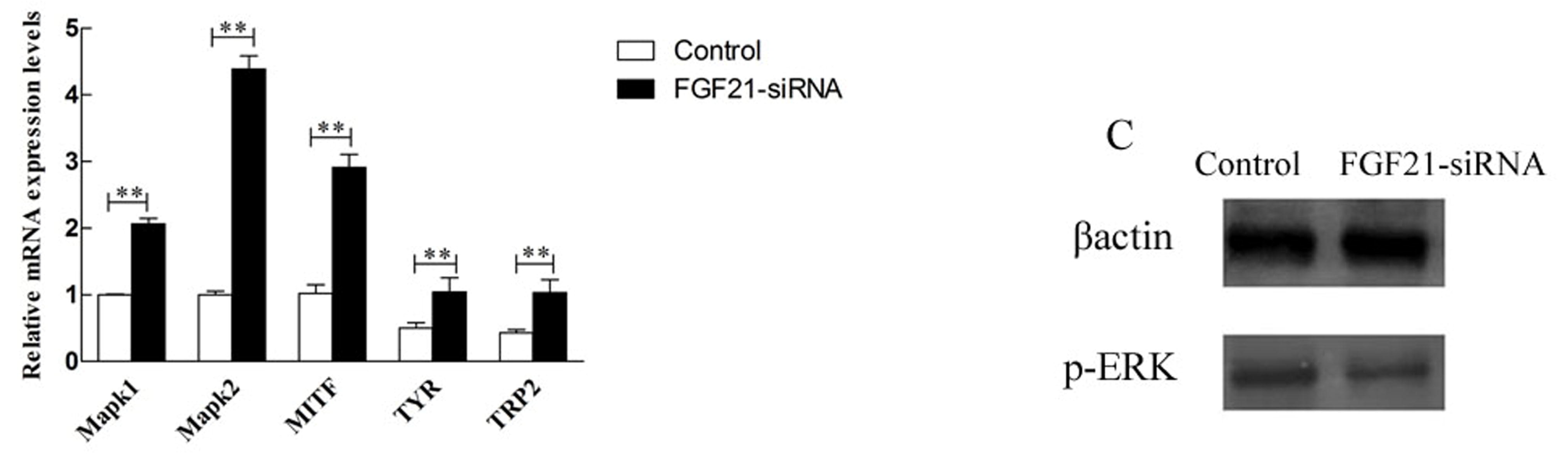

B

MITF

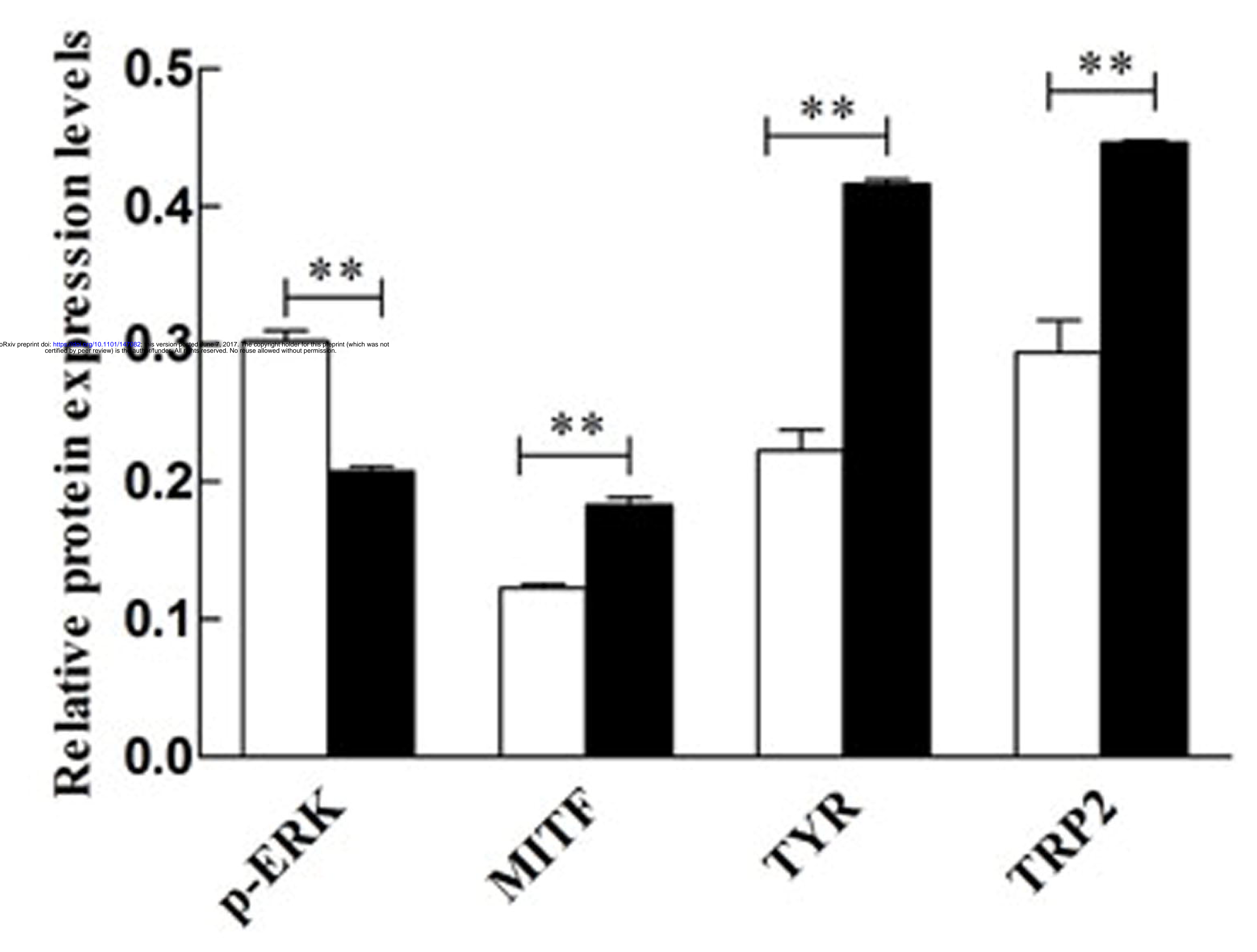

Control

FGF21-siRNA

TYR

TRP2
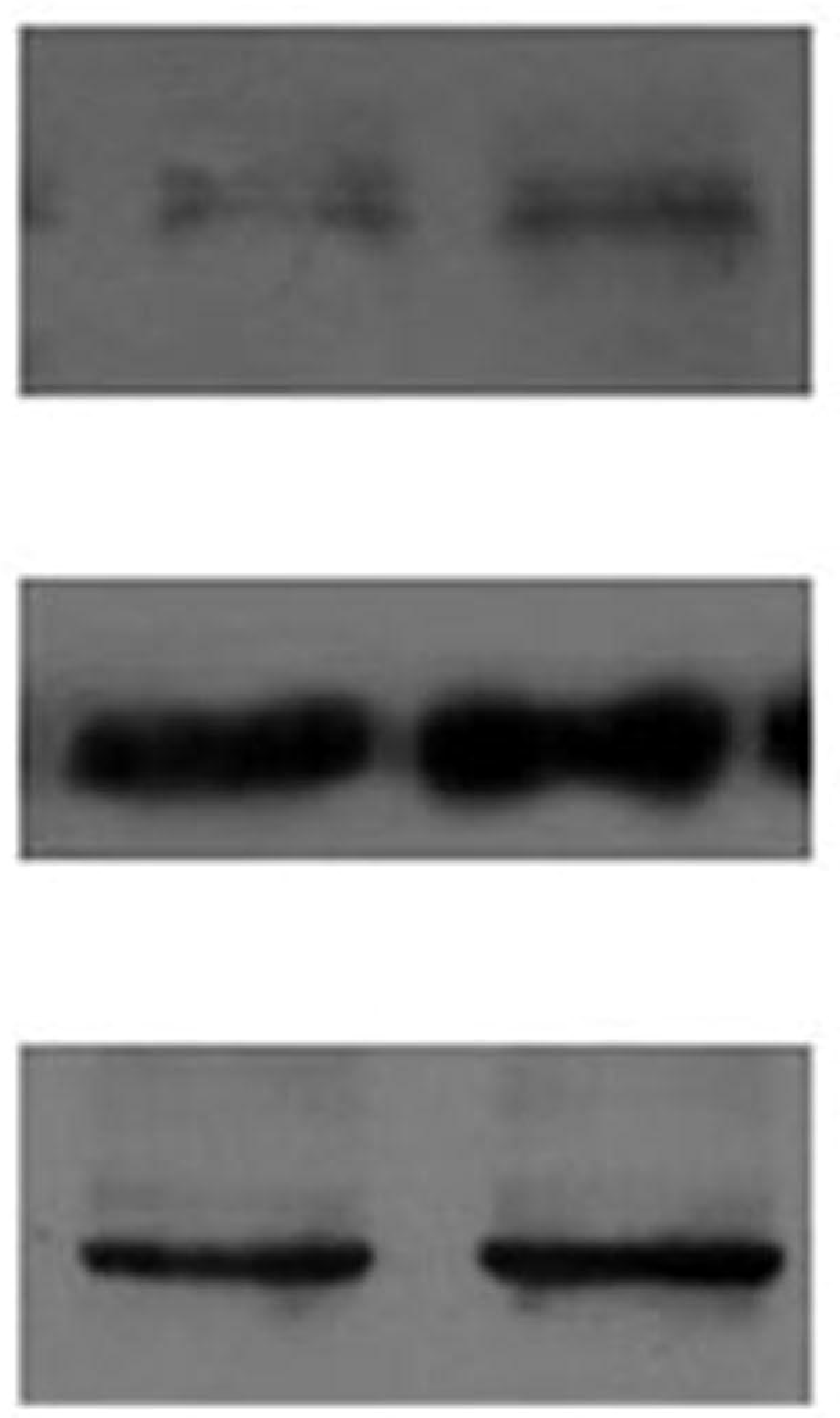
A

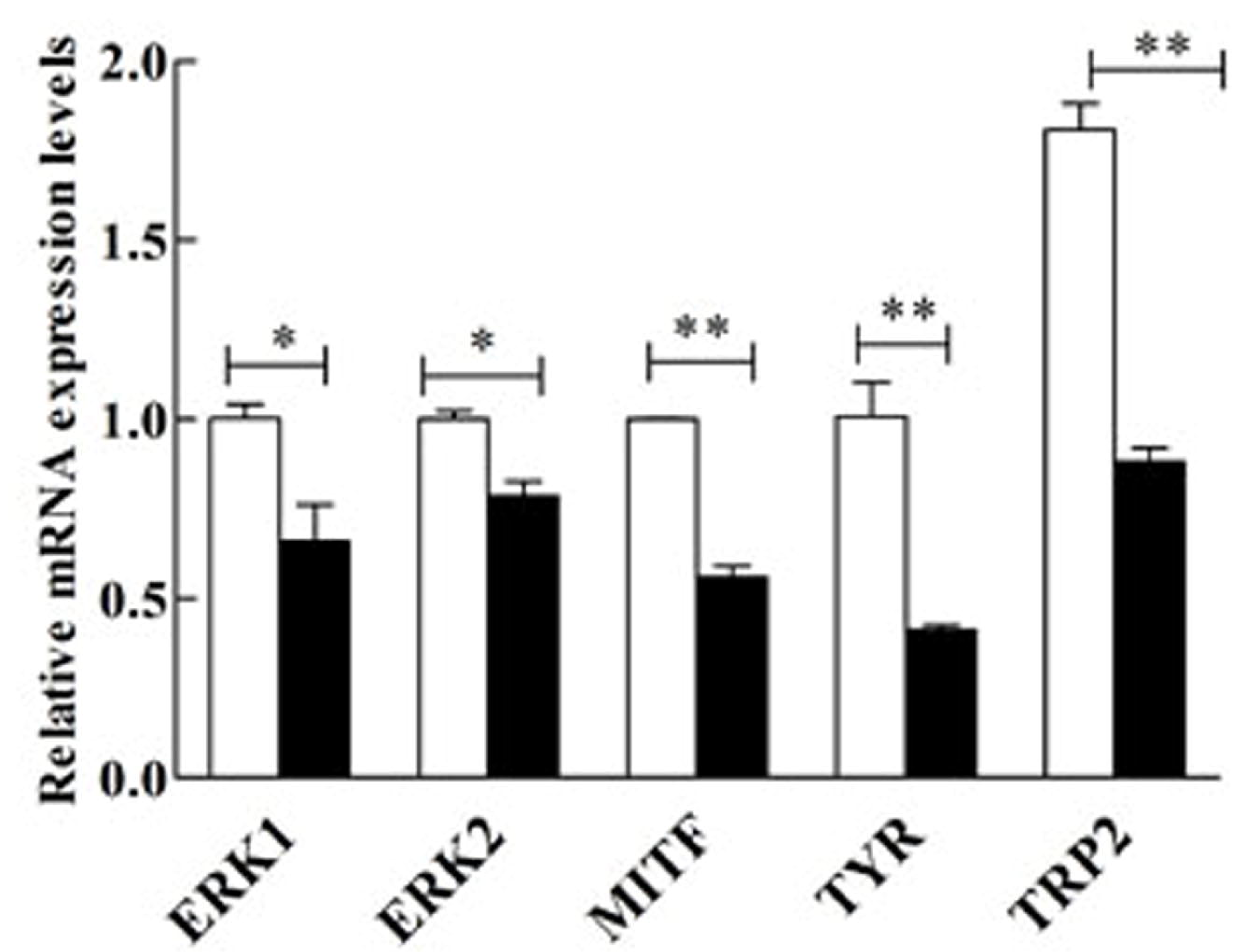

$\square$ Control

- $\mathrm{PMSCV}+\mathrm{FGF} 21$

B

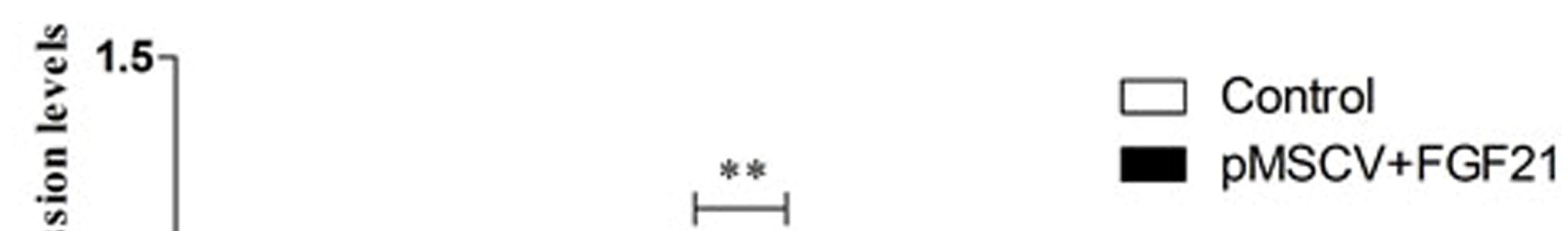

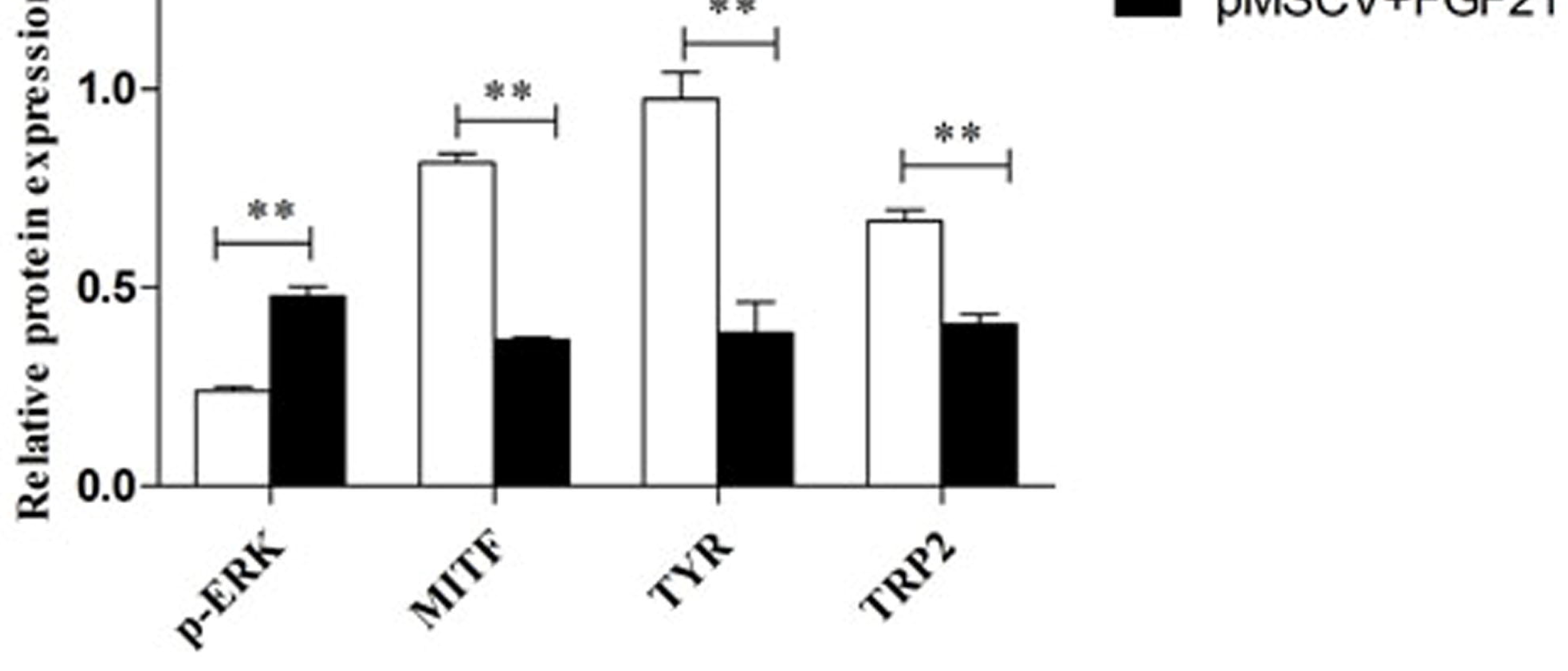

C Control pMSCV+FGF21

$\beta$ actin

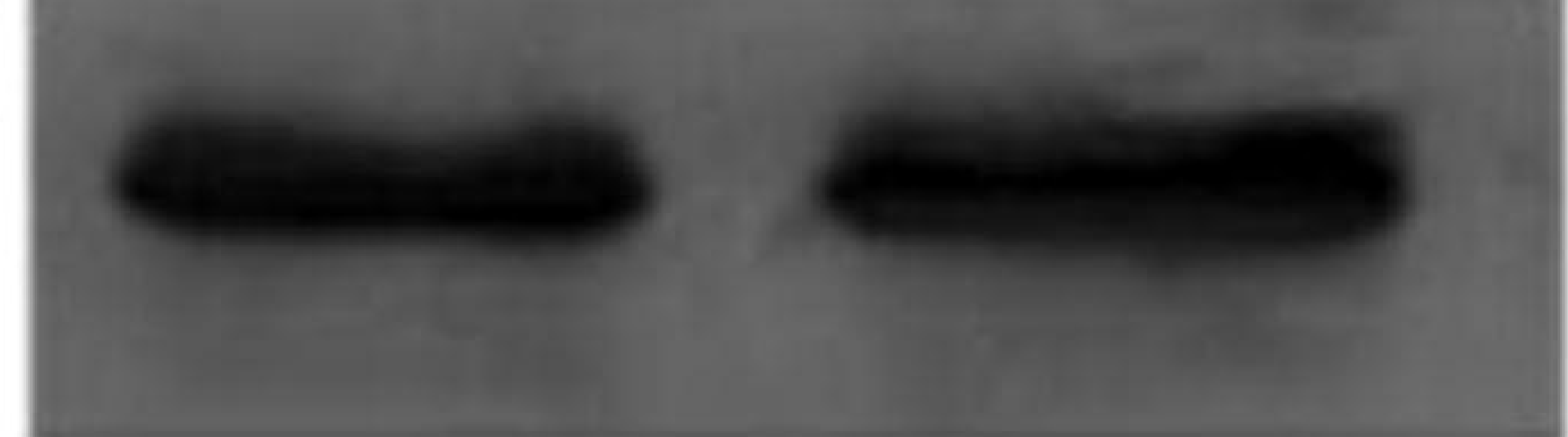

p-ERK

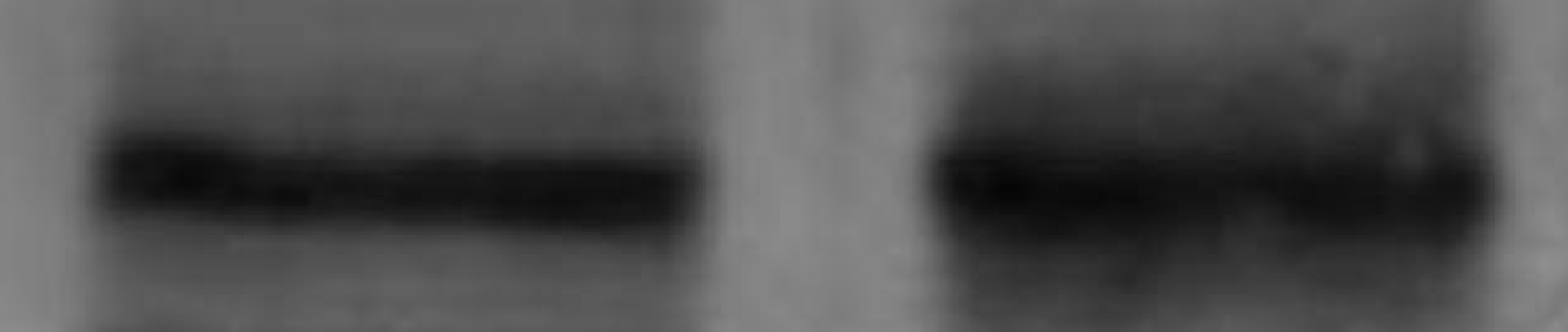

MITF

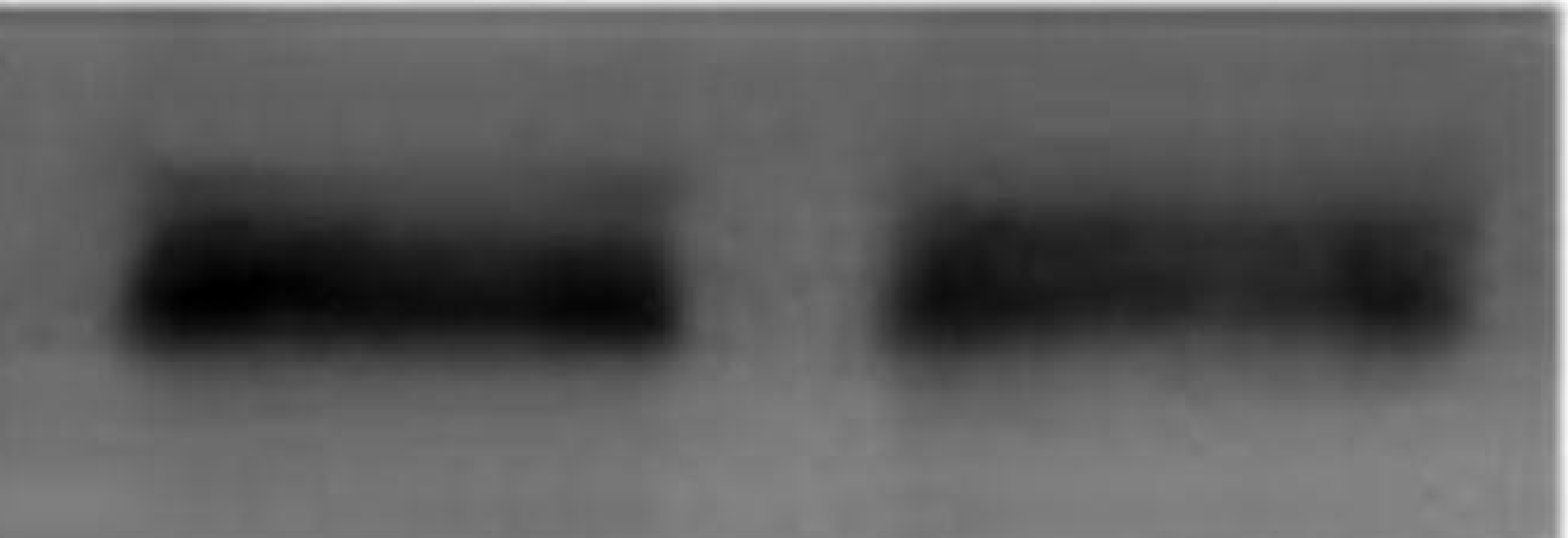

TYR

TRP2
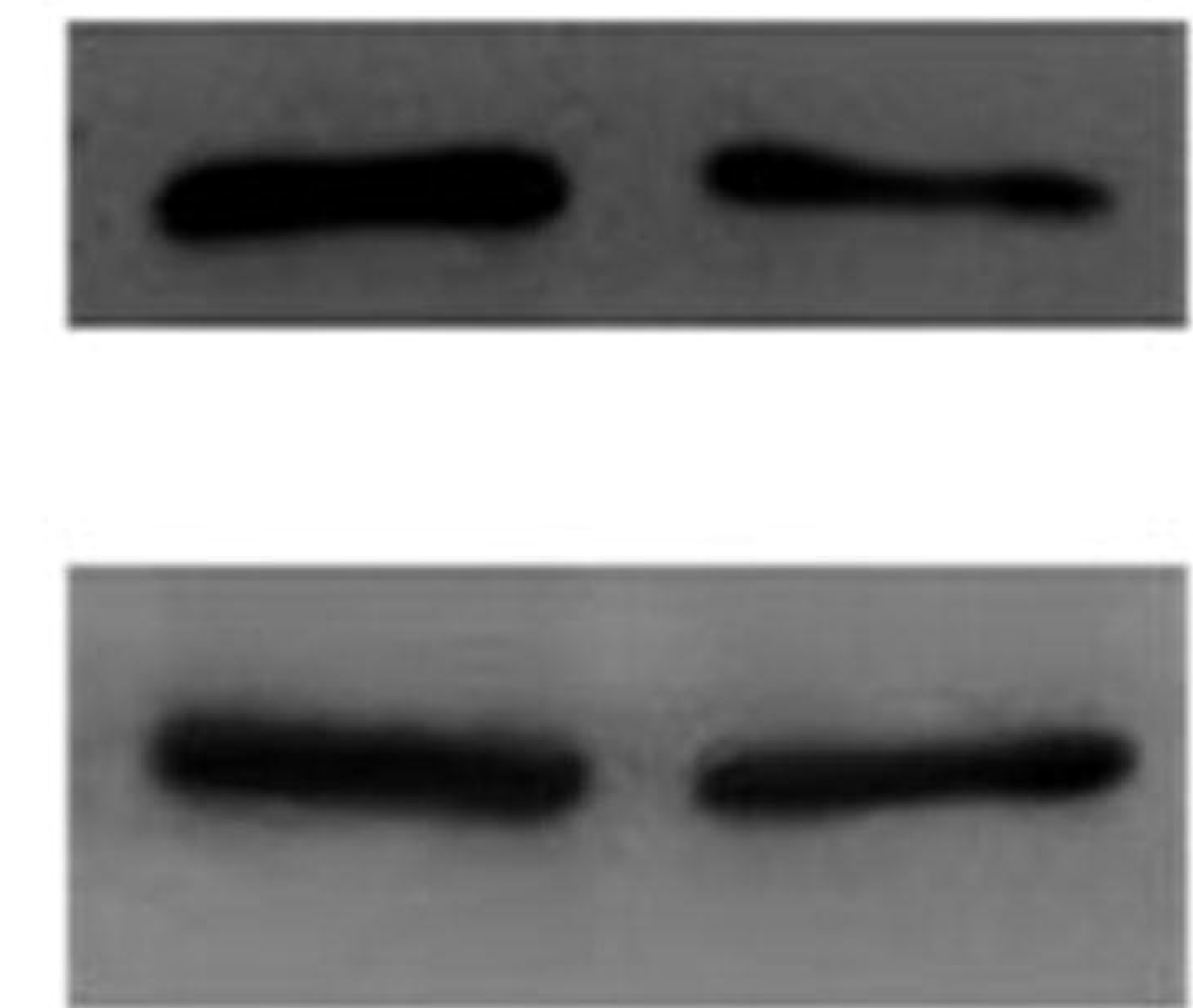